\title{
Boron Determination in Steels by Inductively Coupled Plasma Atomic Emission Spectrometry. Comparative Study of Spark Ablation and Pneumatic Nebulization Sampling Systems*
}

\author{
Aurora G. Coedo, Teresa Dorado, Ester Escudero and Isabel G. Cobo \\ Centro Nacional Investigaciones Metalurgicas, Consejo Superior de Investigaciones Cientificas, Gregorio \\ del Amo 8, 28040 Madrid, Spain
}

\begin{abstract}
An experimental study for the determination of boron in steels by inductively coupled plasma atomic emission spectrometry is presented. A comparison is made of spark ablation and pneumatic nebulization (after microwave digestion) sampling systems. A one-step microwave digestion procedure for total boron content using diluted aqua regia $\left(\mathrm{HCl}+\mathrm{HNO}_{3}, 3+1\right)$ and high pressure vessels was developed. The influence of microwave power and time on the dissolution of boron compounds is discussed. The strongest available conditions $(0 \Omega, 600 \mathrm{~V}, 600 \mathrm{~Hz})$ were required for spark ablation sampling. The stability of spark sampling during the spark ablation-ICP process was tested by plotting iron and boron emission versus sparking time. The iron content of collected and dissolved spark-produced particles was analysed and showed that the amounts of aerosol from different boron steels samples during $90 \mathrm{~s}$ sparking processes were fairly similar. The analytical performance of both systems was evaluated. Using pneumatic nebulization, after microwave digestion of the sample, a detection limit for boron of $2.6 \mu \mathrm{g} \mathrm{g}^{-1}$ and overall relative standard deviation (RSD) values of $1-3.5 \%$ were found. For spark ablation the detection limit for boron was $0.65 \mu \mathrm{g} \mathrm{g}^{-1}$, the overall RSD ranging from 0.5 to 1.5\%. A comparison of the data for British Chemical Standards (BCS) Certified Reference Materials (CRMs) Carbon Steel Residual Series (Group B) and Spectroscopic Standard (SS) 456/1 to 460/1, indicated that the accuracy of both methods was satisfactory.
\end{abstract}

Keywords: Spark ablation; microwave digestion; inductively coupled plasma atomic emission spectrometry; boron determination; steel

The importance of the effect caused by very low concentrations of boron on the physical properties of steels (hot workability, hardenability, creep resistance, etc.), requires precise and accurate determination of this element. Compared with most other alloying elements, the amount of boron added to steel is extremely small and commonly the boron content in boron treated carbon steels ranges from $0.0005 \%$ or less to about $0.005 \%$ (too little boron may be ineffective and too much can cause difficulties in rolling or forging). ${ }^{1}$ Boron was probably the first element in steelmaking to require analytical techniques capable of determining this element at the $\mu \mathrm{g} \mathrm{g}^{-1}$ level.

Ambrose et $a l^{2}$ have reviewed methods for the determination of boron in steel. These workers also examined and commented on the method adopted by the European Committee for Iron and Steel Standardization/Technical Committee 20 (ECISS/TC20), and presented analytical results for the boron content of some certified reference steel samples using different methods. The procedure suggested by Thiering, ${ }^{3}$ and the study mentioned above form the basis of the International Organization for Standardization (ISO) standard method for boron determination. ${ }^{4}$ This international standard is applicable to boron contents in steel of between $0.0005 \%$ and $0.012 \%(\mathrm{~m} / \mathrm{m})$ and specifies a spectrophotometric procedure using curcumin which is sensitive to experimental conditions during the dissolution steps in order to obtain quantitative dissolution of all the boron compounds and low blank values.

The technique of microwave digestion in pressure vessels has progressed significantly over recent years, ${ }^{5-8}$ and offers a very effective and fast dissolution system in addition to low blank values. These characteristics make the system very attractive for use in inductively coupled plasma (ICP) methods.

Over the past several years there has been growing interest in developing direct solid sample introduction

\footnotetext{
*Presented at the 1993 European Winter Conference on Plasma
} Spectrochemistry, Granada, Spain, January 10-15, 1993. systems for use with ICP spectrometry. Broekaert et al. ${ }^{9}$ reported a study of some techniques for direct solid sampling in plasma spectrometry. Although several studies ${ }^{10-13}$ have shown that the spark ablation-ICP combination is a convenient means of direct solid steel sample analysis, it is difficult to ascertain the physical and chemical characteristics of the spark-produced aerosol and consequently the analytical performance of the technique. Watters et al. ${ }^{14}$ presented a detailed study of the physical properties and the chemical composition of a spark-produced aerosol and corresponding erosion craters on brass certified reference materials (CRMs).

In this paper a comparison is made between pneumatic nebulization (from microwave dissolved samples) and spark ablation sampling systems used with ICP spectrometry for analysing boron in steels.

In an effort to establish a microwave dissolution procedure, a study was conducted to select the most important variables conditioning the microwave digestion process: acid mixture, digestion vessels, proportion of sample to reagent required, power, time and number of samples.

For spark ablation the first assays aimed to prove the similarity of the amounts of aerosol produced (from different steel samples) and the stability of its chemical composition. The aerosol particles produced for the sparking process were collected and dissolved in aqua regia $\left(\mathrm{HCl}+\mathrm{HNO}_{3}, 3+1\right)$ and the iron content was analysed by ICP spectrometry to determine the total mass eroded. The stability of the aerosol composition was verified by plotting the boron and the iron emission intensities versus spark erosion time curves.

British Chemical Standards (BCS) CRMs Carbon Steel Residual Series Group B and Spectroscopic Standard (SS) $456 / 1$ to $460 / 1$, were used for testing both methods.

\section{Experimental}

\section{Instrumentation}

Inductively coupled plasma atomic emission spectrometry (ICP-AES) measurements were performed with a Jobin 
Table 1 Selected working parameters

\begin{tabular}{ll}
$\begin{array}{l}\text { Microwave oven } \\
\text { Power/W }\end{array}$ & 360 \\
Time/min & 30 \\
Digestion vessels & HPV 80 \\
Sampling spark & \\
Voltage/V & 600 \\
Repetition rate $(\mathrm{f}) / \mathrm{Hz}$ & 600 \\
Capacitance $/ \mu \mathrm{F}$ & 1 \\
Inductance $/ \mu \mathrm{H}$ & 20 \\
Resistance $(R) / \Omega$ & 0 \\
Gas flow rate $/ 1 \mathrm{~min}^{-1}$ & Permanent carrier gas, 2.1 ; \\
& \multicolumn{1}{c}{ analysis gas, 0.80} \\
ICP-AES & \\
Power $/ \mathrm{W}$ & 950 \\
Gas flow rate $/ 1 \mathrm{~min}^{-1}$ & 14 \\
Observation height $/ \mathrm{mm}$ & 13
\end{tabular}

Yvon JY 24 plasma spectrometer, purging with nitrogen. Spark ablation sampling was carried out with a Jobin Yvon JY-SAS sparking unit. Microwave digestion solutions were obtained using a Milestone MLS-1200 microwave oven and HPV 80 high pressure vessels. Table 1 lists the selected operating conditions.

\section{Samples}

British Chemical Standard CRMs Carbon Steel Residual Series BCS-CRM and SS $456 / 1$ to $460 / 1$, are boron-carbon steels with certificate boron contents between 0.0015 and $0.0118 \%$. The BCS-CRM samples come in chip form for wet analysis, while SS samples come in disc form for direct solid analysis. The boron content values are similar in both types of sample and they were re-certified in 1988, in accordance with the study conducted by the European Committee for Iron and Steel Standardization (ECISS/TC 20). The Fe-O sample, with a boron content below $1 \mathrm{ppm}$, was employed as a 'blank'.

\section{Results and Discussion}

\section{Microwave digestion}

Boron is present in steel both in acid-soluble and nonsoluble forms. The most significant non-soluble boron compound is $\mathrm{BN}$. The dissolution step not only increases analysis time, but can also cause errors when ICP-AES is used by increasing the spectral background and decreasing overall analytical sensitivity.

In this paper a microwave digestion system was used to completely dissolve the sample. Tests were performed on different acid mixtures (containing different proportions of hydrochloric, nitric, hydrofluoric, phosphoric and perchloric acids) and different proportions of samples and reagents to find the simplest procedure for stable and quantitative dissolution of all types of boron compounds.

After this selection different microwave digestion programmes were applied varying the operational parameters of the oven (microwave power and time). Two types of pressure vessels were used: low pressure SV-140 $\left[p_{\max }=18\right.$ bar $\left.\left(1 \mathrm{bar}=1 \times 10^{5} \mathrm{~Pa}\right), V=140 \mathrm{ml}\right]$ and high pressure HPV $80\left(p_{\max }=150\right.$ bar, $\left.V=80 \mathrm{ml}\right)$. When a closed vessel is used, the pressure rises with an increase in microwave power and depending on how long the microwave acts. Consequently the temperature of the digestion mixture increases also, enhancing efficiency.

The BCS $460 / 1$ sample was used to test the influence of the parameters studied. Coedo and Dorado ${ }^{15}$ concluded that by using an ICP method, this CRM with a certificate value for total boron of $28 \mathrm{ppm}$ contains a high proportion

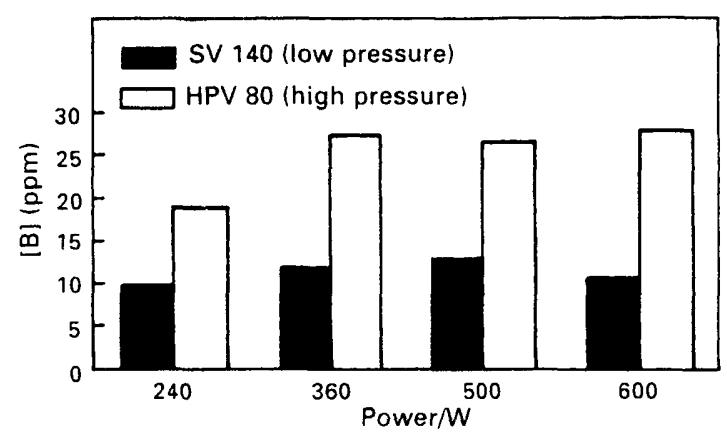

Fig. 1 Effect of microwave power on boron dissolution of BCSCRM 460/1 for two types of pressure vessel for a time of $30 \mathrm{~min}$

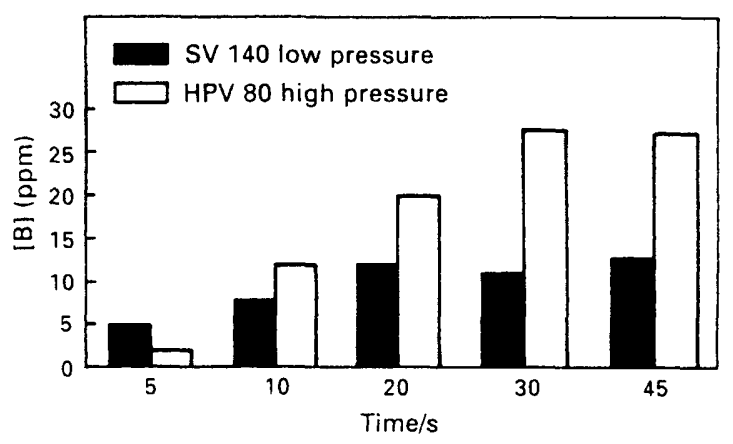

Fig. 2 Effect of time on boron dissolution of BCS-CRM 460/1 for two types of pressure vessel. Power, $600 \mathrm{~W}$ for S V 140 and $360 \mathrm{~W}$ for HPV 80

of non-soluble boron particles $(\approx 53 \%$ of the total boron content) and so can be used to evaluate the efficiency of the dissolution procedures.

The results obtained by varying the microwave power for the two types of vessels and for a fixed period of $30 \mathrm{~min}$ are shown in Fig. 1.

The influence of time for a fixed power value is shown in Fig. 2. This power value was set at $600 \mathrm{~W}$ for SV-140 (the maximum value that could be applied with the selected reagents without risking vessel failure) and at $360 \mathrm{~W}$ for HPV-80 (this level of power was enough to recover all the boron content when this type of vessel was used)

The BCS $460 / 1$ sample was completely dissolved only when high pressure vessels were used. The volume of the high pressure vessels (HPV 80 ) is $80 \mathrm{ml}$, the volume of the low pressure vessels (SV 140) being $140 \mathrm{ml}$. This difference means that under the same operating conditions (same portion of test sample, reagents and oven parameters) the pressure reached in the HPV vessels is greater and consequently the temperature and the dissolution process efficiency is higher. It could be suggested that a high pressure is required to dissolve all the boron compounds in sample BCS 460/1 owing to the presence of a high amount of boron nitride and of a high carbon content $(0.45 \%)$ producing fine carbon particles that help to retain other products such as boron compounds.

As a result of this study, the following operating dissolution procedure was adopted. A $0.250 \mathrm{~g}$ sample was dissolved in an HPV-80 vessel (maximum pressure $150 \mathrm{bar}$ ) with $5 \mathrm{ml}$ of $\mathrm{HCl}+2 \mathrm{ml}$ of $\mathrm{HNO}_{3}+10 \mathrm{ml}$ of $\mathrm{H}_{2} \mathrm{O}+2$ drops of $\mathrm{HF}$, by applying a one-step microwave programme of $360 \mathrm{~W}$ lasting $30 \mathrm{~min}$. After cooling, the solutions obtained were made up to $50 \mathrm{ml}$ with water, in graduated polyethylene flasks. The efficiency of the method was verified by simultaneous treatment of 2-6 samples. 


\section{Spark Ablation}

Since the use of the spark to generate sample aerosol divides the sampling and excitation processes into two separate events, systematic errors in each step should be examined in order to evaluate the results provided by the combined spark ablation-ICP technique. Most studies have centred on the erosion of the sample surface, yet the analytical information is carried to the plasma by the particles that are formed. A high sample ablation and rate of analyte introduction into the plasma provide higher levels of sensitivity and higher detection capacity. The amount of analyte reaching the plasma increases both with the voltage and the repetition rate. However, the particle size is increased by raising the voltage applied, not by raising the repetition rate. $A$ high repetition rate is preferable to a high voltage because of the particle size produced. An increase in the particle size results both in decreased stability and poor signal-to-background ratio of ICP signals (the finer the particles, the better the stability in the plasma). Consequently, source parameters resistance $(R)$, voltage $(V)$ and repetition rate $(f)$, which have a clear influence on the analyte produced, must be changed in line with the nature and characteristics of the samples to be analysed. The condition and hence the burn is stronger with decreasing $R$, but increasing $V$ or $f$. Owing to the hardenability of boron steels and considering that $f$ and $R$ have a direct influence on the amount of material eroded but not on the particle size, these parameters were set to obtain strong burns $(R=0$ $\Omega$ and $f=600 \mathrm{~Hz}$ ). Tests were conducted using different voltage spark values, from 350 to $600 \mathrm{~V}$ (maximum available voltage). The most repeatable and accurate results were obtained by applying the strongest conditions $(600 \mathrm{~V})$. As a result of this study, the operating conditions listed in Table 1 were selected for spark sampling.

As the samples heated up during the sparking process, they were cooled in a carbon dioxide stream to allow consecutive measurements to be made under the same conditions, with a view to achieving better sampling repeatability.

Under the established conditions, a spark ablation-ICP process lasted about $85 \mathrm{~s}$ (30 s for pre-integration and cleaning, $15 \mathrm{~s}$ for transfer and $40 \mathrm{~s}$ for a 'three-point' mode measurement with background correction). To minimize sparking times, peak intensities were measured employing a 'three-point' mode. In this mode the window includes three points with a distance between them of $0.0003 \mathrm{~nm}$, and the intensity value is a weighted average of these three points.

The aerosols produced from $90 \mathrm{~s}$ spark ablation attacks of boron-carbon steels were trapped and dissolved in diluted aqua regia. These particles were removed from the end of the $0.75 \mathrm{~m}$ long tube $(5 \mathrm{~mm}$ i.d.), which transports the analyte from the sparking chamber to the ICP torch, with the aid of an argon gas flow of $0.81 \mathrm{~min}^{-1}$, by introducing

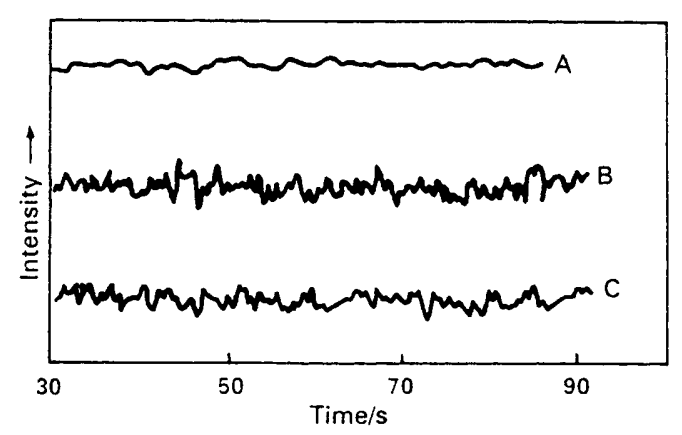

Fig. 3 Effect of sparking time on emission intensity of: A, Fe; B, B using SS 459/1; and C, B using SS 456/1 the end of the tube into the acid solution $(10 \mathrm{ml}$ of aqua regia $+40 \mathrm{ml}$ of water). After completing the dissolution by heating, the iron content of these solutions was determined by ICP-AES in order to calculate the amount of analyte produced during the period of time from the beginning of the spark process to the acquisition of a spark ICP measurement $(\approx 85 \mathrm{~s})$. The results show good repeatability of the amount of eroded material produced during sparking processes from the different boron-carbon steel samples tested. This amount was $0.700 \pm 0.025 \mathrm{mg}$ of iron for sparking periods of $90 \mathrm{~s}$.

The profiles of the boron and the iron emission intensities versus spark time show the signal stability of the aerosol produced under the selected spark conditions, at least for the duration of the spark ablation-ICP measurement (Fig. 3).

\section{ICP Measurements}

Before measurement, a preliminary study was conducted in order to choose the boron analytical line. Under the operating parameters selected for the JY 24 ICP instrumentation, the lowest detection limit and the best values of relative standard deviation (RSD) were obtained for the line at $208.959 \mathrm{~nm}$

The scans around the selected boron line are shown in Fig. 4. These scans correspond to the 'blanks' and to the calibration samples employed with both sampling systems. After having considered these scans, values for background correction were measured at $208.925 \mathrm{~nm}(0.035 \mathrm{~nm}$ left of the boron peak). By using pneumatic nebulization, blank values measured with background correction were approximately half the total emission intensity. There are two components in the blank total emission, a specific peak signal and an elevation of the background.

Table 2 gives the analytical performance expressed in terms of background equivalent concentration (BEC), detection limit (DL) and precision.

To calculate the DL (defined as the concentration of a solution which gives an absorbance equal to three times the $\mathrm{SD}$ of the blank) the following formula was used:

$$
D L=\frac{3 \times R S D_{\text {background }} \times B E C}{100}
$$

The BEC values (boron concentration giving a net analyte signal equal to the background signal) were $138 \mu \mathrm{g} \mathrm{g}^{-1}$ for pneumatic nebulization and $46 \mu \mathrm{g} \mathrm{g}^{-1}$ for spark ablation. In the first system the BEC value was so high because of the

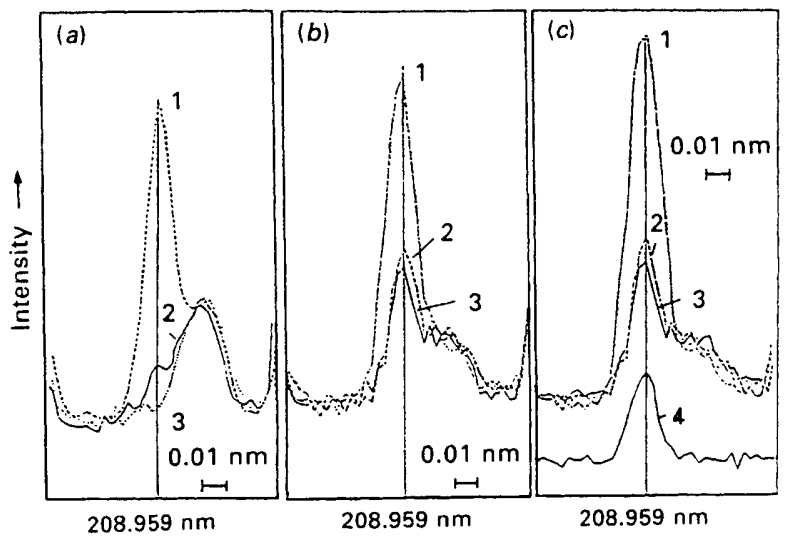

Fig. 4 Emission profiles. (a) Spark ablation: 1, SS 459/1; 2, SS $456 / 1$; and $3, \mathrm{Fe}-\mathrm{O}$. (b) Pneumatic nebulization $(0.5 \mathrm{~g}$ per $100 \mathrm{ml})$ : 1, BCS-CRM 459/1; 2, BCS-CRM 456/1; and 3, Fe-O. (c) Solutions (100 ml): $1,0.5 \mathrm{~g}$ of Fe plus $0.7 \mathrm{ppm}$ of $\mathrm{B} ; 2,0.5 \mathrm{~g}$ of Fe plus 0.1 ppm of B; 3, $0.5 \mathrm{~g}$ of Fe; and 4, blank 
Table 2 Certified and found values for SS samples: $x=$ mean of six determinations; $T=$ interval of uncertainty of the mean, for a level of probability of $95 \%$. $T=t_{95,5} \sqrt{\alpha_{x}^{2} / 5}$ where $t_{95,5}=2.571$

Total $\mathrm{B} / \mu \mathrm{g} \mathrm{g}^{-1}$

\begin{tabular}{|c|c|c|c|c|c|c|c|}
\hline \multirow[b]{2}{*}{ Samples } & \multirow[b]{2}{*}{ Certified } & \multicolumn{3}{|c|}{ Microwave digestion } & \multicolumn{3}{|c|}{ Spark ablation } \\
\hline & & $x$ & $T$ & $\alpha$ & $x$ & $T$ & $\alpha$ \\
\hline SS $456 / 1$ & 15 & 14.7 & 0.31 & 0.26 & \multicolumn{3}{|c|}{ Calibration $\mathrm{L}^{*}$} \\
\hline SS $457 / 1$ & 25 & 24.4 & 0.50 & 0.43 & 25.3 & 0.90 & 0.78 \\
\hline SS $458 / 1$ & 61 & 59.6 & 0.81 & 0.70 & 62.0 & 1.12 & 0.97 \\
\hline SS $459 / 1$ & 118 & 120.0 & 1.32 & 1.14 & \multicolumn{3}{|c|}{ Calibration $\mathrm{H} \dagger$} \\
\hline SS $460 / 1$ & 28 & 26.8 & 0.49 & 0.42 & 27.3 & 0.85 & 0.74 \\
\hline CENIM & - & 49.5 & 0.47 & 0.41 & 48.7 & 0.90 & 0.78 \\
\hline
\end{tabular}

* $\mathrm{L}=$ low calibration standard.

$+\mathrm{H}=$ high calibration standard.

background elevation produced by the iron matrix and of the elevation of the specific signal as a result of the dissolution process (as can be seen in Fig. 4). At levels five times higher than the corresponding DLs, RSDs $(n=6)$ of 1.3 and $3.5 \%$ were obtained using pneumatic nebulization and spark ablation, respectively.

\section{Calibration}

After verifying the linearity of the boron emission intensities versus boron concentrations, within the interval of boron contents considered, only two samples ('low' and 'high') were used to obtain the calibration graphs. Using pneumatic nebulization, two calibration samples were prepared with 0.01 and $0.07 \mathrm{mg}$ of boron, added from a standard boron solution, in the presence of $0.5 \mathrm{~g}$ of pure iron (Fe-O sample) per $100 \mathrm{ml}$. These two calibration solutions are equivalent to steel samples containing 0.0020 and $0.014 \%$ of boron, respectively.

Two samples were used for calibration with spark ablation: sample SS $456 / 1$ ( $0.0015 \%$ of boron) as 'standard low' and sample SS $459 / 1$ ( $0.0118 \%$ of boron) as 'standard high'.

The ICP calibration graphs obtained from pneumatic nebulization and spark ablation sampling systems are shown in Fig. 5. In these graphs, the BCS-CRM and SS samples not used for calibration are interpolated by plotting their emission values against the certified concentrations. The correlation coefficients obtained with the linear regression of all the data points were 0.9992 and of 0.9990 , respectively.

The results obtained from the above calibration graphs for the BCS-CRM and SS samples series and for a Centro Nacional de Investigaciones Metalúrgicas (CENIM) sample are shown in Table 3 . The mean values obtained with both methods for the boron content in the CENIM sample were compared by employing the Student's $t$-test. This sample

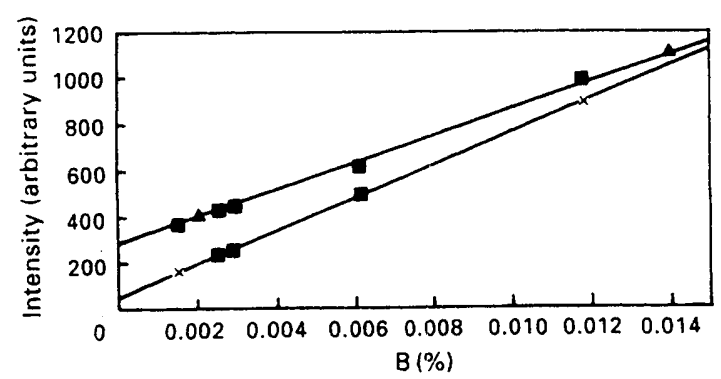

Fig. 5 Calibration graphs obtained using: $\boldsymbol{\Delta}$, pneumatic nebulization; $x$, spark ablation; and $\square$, BSC-CRM (pneumatic nebulization) and SS (spark ablation) of samples was analysed first in disc form by spark ablation sampling and then by pneumatic nebulization of a dissolution of chips obtained from the disc sample.

As the same number of determinations were carried out with the two methods $(n=6)$, mutual agreement was tested by the ratio

$$
t=\frac{x_{\mathrm{A}}-x_{\mathrm{B}}}{\sqrt{\frac{\sigma_{\mathrm{A}}^{2}+\sigma_{\mathrm{B}}^{2}}{n-1}}}
$$

where $x=$ mean; $\sigma=\mathrm{SD} ; \nu=$ degrees of freedom; $\operatorname{ad}(1-\alpha)$ is the probability level. For a probability level $(1-\alpha)=0.95$ and 10 degrees of freedom $(\nu=2 n-2=10)$, in Student's tables $t=2.228$. The value calculated from figures in Table $3, t=2.026$, is lower than the tabulated one. This means that the difference between the two means is statistically insignificant and can be explained by random errors alone.

\section{Conclusion}

The high pressure microwave digestion method is a valid system for complete dissolution of boron compounds in steels. The sample dissolution time is reduced from hours to $30 \mathrm{~min}$. A further advantage was seen when six samples were dissolved simultaneously. As no evaporation occurred during the digestion process, only a few millimetres of aqua regia were required as digestion reagent, leading to low blank values. This allows lower DL to be attained than with conventional dissolution procedures. As a result of reduced sample handling and the impossibility of airborne contamination, the risk of contamination is substantially reduced.

By employing strong spark conditions $(0 \Omega, 600 \mathrm{~V}, 600$ $\mathrm{Hz}$ ), spark ablation can be used as a solid sampling system for the determination of boron in steels. The amounts of spark aerosol produced from boron steel surfaces prepared under the same conditions, during a spark ablation ICP process, are fairly similar and their elemental composition ( $\mathrm{B}$ and $\mathrm{Fe}$ ) remains stable and matches the bulk composition reasonably well.

Table 3 Analytical performance

\begin{tabular}{|c|c|c|c|}
\hline Sampling system & $\mathrm{BEC}^{*} / \mu \mathrm{g} \mathrm{g}^{-1}$ & $\mathrm{DL}+/ \mu \mathrm{g} \mathrm{g}^{-1}$ & RSD \\
\hline $\begin{array}{l}\text { Microwave digestion } \\
(0.5 \mathrm{~g} \text { per } 100 \mathrm{ml}) \\
\text { Spark ablation }\end{array}$ & $\begin{array}{r}138 \\
46\end{array}$ & & $\begin{array}{l}1.3 \\
3.5\end{array}$ \\
\hline \multicolumn{4}{|c|}{$\begin{array}{l}\text { * Background equivalent concentration giving an emission signal } \\
\text { equal to twice the total emission of the blank. } \\
\dagger \text { Detection limit producing an emission signal equal to three } \\
\text { times the SD of the blank, measured with background correction. } \\
\ddagger \text { Relative standard deviation }(n=6) \text { at five times the DL. }\end{array}$} \\
\hline
\end{tabular}


The ICP-AES technique with the two tested sampling systems could replace traditional chemical methods for boron analysis in steel samples. The drawback to the microwave digestion method is its higher background values and, consequently, its lower signal-to-background ratios and higher DL (2.5 times that achieved with spark ablation), however, the precision values are better. The main obstacle to spark ablation sampling is the need to use solid standard samples for calibration.

The accuracy of both methods is demonstrated by the results obtained for the CRMs analysed.

The assays concerning development of the microwave sample procedure form part of a research project financially supported by the European Community for Steel and Carbon (ECSC), No. 7210/GD/936 (E2. 3/89).

\section{References}

1 Steel and Its Heat Treatment, ed. Thelning, K. E., Butterworths, London, 2nd edn., 1984, p. 405.

2 Ambrose, A. D., Harine, M., Staats, G., and Weichert, E., Steel Res., 1989, 60, 363.

3 Thierig, D., Fresenius' Z. Anal. Chem., 1982, 310, 154.
4 ISO 10153, 1991. International Organization for Standardization, P.O. Box 56, CH-1211, Geneva 20, Switzerland.

5 Introduction to Microwave Sample Preparation, eds. Kingston, H. M., and Jassie, L. B., ACS Professional Reference Book, Washington D.C., 1988.

6 Kammin, W. R., and Brandt, M. J., Spectrosc. Int., 1989, 1, 50

7 Gilman, L. B., and Engelhart, G., Spectrosc. Int., 1990, 2, 16.

8 Progress of Analytical Chemistry in the Iron and Steel Industry, Commission of the European Communities. EUR 14113, Luxembourg, 1992, pp. 293-295.

9 Broekaert, J. A. C., Leis, F., Raeymaekers, B., and Zaray, Gy., Spectrochim. Acta, 1988, 39, 339.

10 Mandoki, A., Boron in Low Alloy Steels; Analytical Report. Instruments S.A., Jobin Yvon, Longjumeau, 1991.

11 Lemarchand, A., Labarraque, G., Masson, P., and Broekaert, J. A. C., J. Anal. At. Spectrom., 1987, 2, 481.

12 Prell, L. J., and Koirtyohann, S. R., Appl. Spectrosc., 1988, 42, 1221 .

13 Coedo, A. G., Dorado, L. T., Seco, J. L., and Cobo, I. G., J. Anal. At. Spectrom., 1992, 7, 11.

14 Watters, R. L., Jr., DeVoe, J. R., Shen, F. H., Small, J. A., and Marirenko, R. B., Anal. Chem., 1989, 61, 1826.

15 Coedo, A. G., and Dorado, L., Rev. Metal. Madrid, 1985, 21 , 87.

Paper 2/05430B

Received November 9, 1992 Accepted February 8, 1993 